\title{
Association Between Early Markers of Renal Injury and Type 2 Diabetic Peripheral Neuropathy
}

\author{
Zhi Yang ${ }^{1, *}$ \\ Xiaoyang Lou ${ }^{1,2, *}$ \\ Jie Zhang ${ }^{1,3, *}$ \\ Ronghui Nie ${ }^{1,3}$ \\ Jiang Liu' \\ Ping Tu' \\ Peng Duan' \\ 'Department of Endocrinology and \\ Metabolism, The Third Hospital of \\ Nanchang, Nanchang, 330000, Jiangxi, \\ People's Republic of China; ${ }^{2}$ Jiangxi \\ University of Traditional Chinese \\ Medicine, Nanchang, 330004, Jiangxi, \\ People's Republic of China; ${ }^{3}$ Jiangxi \\ Medical College of Nanchang University, \\ Nanchang, 330036, Jiangxi, People's \\ Republic of China
}

*These authors contributed equally to this work
Correspondence: Peng Duan Department of Endocrinology and Metabolism, The Third Hospital of Nanchang, Nanchang, 330000, Jiangxi,

People's Republic of China

Tel +86 I3479IIII77

Email13479IIII77@I63.com
Objective: Diabetic kidney disease (DKD) and diabetic peripheral neuropathy (DPN) are both common chronic complications of type 2 diabetes mellitus (T2DM). The aim of this study was to examine whether some markers of early renal injury were associated with DPN. Methods: Retrospective hospitalization data from 471 patients with T2DM were analyzed. Subjects were divided into DPN group and non-DPN group according to clinical history, symptoms, signs and nerve conduction study. Markers of glomerular injury [urinary albumin/ creatinine ratio (UACR) and estimated glomerular filtration rate (eGFR)] and tubular injury [urinary $\mathrm{N}$-acetyl- $\beta$-D-glucosaminidase/creatinine ratio $(\mathrm{NAG} / \mathrm{Cr}$ ) and urinary $\beta 2$ microglobulin $(\beta 2-\mathrm{MG})]$ were innovatively combined to assess the association with DPN. Staging of chronic kidney disease (CKD) was classified as G1, G2, G3a, G3b, G4 based on eGFR categories of $\geq 90,60-89,45-59,30-44,15-29 \mathrm{~mL} / \mathrm{min} / 1.73 \mathrm{~m}^{2}$.

Results: DPN was detected in $71.1 \%$ of our population. Subjects in DPN group suffered from older age, longer duration of diabetes and worse blood glucose control compared with non-DPN group. The levels of UACR, NAG/Cr and $\beta 2-\mathrm{MG}$ were significantly increased in DPN group than those in non-DPN group, while eGFR was decreased. The prevalence of DPN increased gradually in G1, G2 and G3-4 of CKD, which were $66.3 \%, 73.2 \%$ and $82.7 \%$ ( $\mathrm{P}=0.014$ ). After adjusting for confounding factors, $\mathrm{NAG} / \mathrm{Cr}>1.41 \mathrm{U} / \mathrm{mmol}$ (the highest tertile) was a consistently independent risk factor for DPN [odds ratio, OR $(95 \%$ confidence interval, CI) $=1.86$ (1.04-3.33)]. However, UACR, eGFR and $\beta 2-\mathrm{MG}$ did not significantly affect the risk of DPN.

Conclusion: When T2DM patients suffer from CKD, DPN will be more likely to appear, accelerate or deteriorate. Some easily available urinary markers of glomerular and tubular damage can be used for early prediction of DPN, in which increased $\mathrm{NAG} / \mathrm{Cr}$ is an independent risk factor for DPN.

Keywords: renal injury, peripheral neuropathy, type 2 diabetes mellitus

\section{Introduction}

Type 2 diabetes mellitus (T2DM) is a common chronic and systemic metabolic disease, which is on the rise year by year. The various chronic complications caused by T2DM significantly increase the disability rate and mortality rate of patients, and diabetes mellitus becomes the ninth major cause of death. ${ }^{1}$ Therefore, early diagnosis and treatment of chronic complications of T2DM are particularly important.

Diabetic peripheral neuropathy (DPN) is one of the microvascular complications of T2DM and has diverse clinical manifestations. Chronic distal symmetric polyneuropathy (DSPN) is the most common type and may be present in at least 10$15 \%$ of newly diagnosed diabetic patients. The growth rate will reach to $50 \%$ after 10 years of disease duration. At present, the diagnosis of DPN comprehensively 
depends on combining clinical history, symptoms, signs and the nerve conduction study (NCS). However, up to $50 \%$ of the patients may be asymptomatic in the early stage of DPN, a delayed diagnosis may only be made on examination or, in some serious cases, such as a painless foot ulcer. ${ }^{2}$ Therefore, innovative methods of early recognition of DPN have some possibility to make.

Diabetic kidney disease (DKD) is another common microvascular complication of T2DM, and occurs in 20$40 \%$ of the patients. ${ }^{3}$ DKD is actually defined as chronic kidney disease (CKD) attributed to diabetes. The diagnosis is based on the presence of albuminuria and/or reduced estimated glomerular filtration rate (eGFR). As is known, peripheral neuropathy is not only the complication of T2DM but also occurs in CKD, independent of diabetes. ${ }^{4}$ In $\mathrm{CKD}$, peripheral neuropathy affects approximately $70 \%$ of the predialysis patients. Dual exposure to diabetes and $\mathrm{CKD}$ is regarded as a contribution for a more rapid onset of nerve injury in patients. ${ }^{5}$

Based on the close connection between peripheral neuropathy and kidney disease, we hypothesize that some well-known markers of renal injury can be used to predict DPN early. A previous study reported that elevated urinary albumin/creatinine ratio (UACR) and decreased eGFR, markers of glomerular injury, might be predictive factors of DPN. ${ }^{6}$ In recent years, markers of renal tubular injury such as urinary $\mathrm{N}$-acetyl- $\beta$-D-glucosaminidase/creatinine ratio (NAG/Cr) and urinary $\beta 2$ microglobulin ( $\beta 2-\mathrm{MG})$ also played an important role in the early prediction of kidney disease. ${ }^{7,8}$ The related effects of these on DPN were rarely explored. Therefore, we innovatively combined markers of glomerular and tubular injury and discussed whether these markers, detected by routine body fluid, could be earlier warning signals for DPN.

\section{Methods}

\section{Study Population}

The subjects of the study were patients with type 2 diabetes who were routinely hospitalized in the department of Endocrinology and Metabolism from the Third Hospital of Nanchang during February 2018 to November 2020. Inclusion criteria were as follows: 1) subjects aged 18 years and above; 2) patients with type 2 diabetes; 3) subjects voluntarily participated in the study and signed written informed consent form at admission. Exclusion criteria were as follows: 1) other types of diabetes; 2) patients with a history of hypothalamus or pituitary disease, acute complications of T2DM, history of cerebral infarction, vitamin B12 deficiency, malignant tumor, degenerative changes of lumbar or cervical spine, pregnancy or lactation; 3) history of severe kidney disease (such as acute kidney injury, dialysis, kidney transplant) or confirmed liver, heart or other organs with seriously damaged; 4) urinary tract infection, acute infection and taking drugs affecting urinary protein; 5) patients hospitalized repeatedly. A total of 476 patients were enrolled, we retrospectively analyzed the hospitalization data of all subjects. However, another five patients failed to enroll because the eGFR of them were less than $15 \mathrm{~mL} /$ $\min / 1.73 \mathrm{~m}^{2}$ which meant they had reached stage 5 CKD. ${ }^{9}$ At last, 471 subjects, 252 men and 219 women, with a mean age of $61.99 \pm 11.88$ years and a mean duration of diabetes of $8.20 \pm 6.74$ years were included in this study.

\section{Nerve Conduction Study}

All subjects accepted NCS (Viking IV EMG system, Nicolet Biomedical, USA) by two experienced technicians, which contained motor nerve conduction studies (median, ulnar, tibial and common peroneal nerves) and sensory nerve conduction studies (median, ulnar, superficial peroneal and sural nerves). Distal motor latency (DML), motor nerve conduction velocity (MCV), compound muscle action potential (CMAP) wave amplitude and distal sensory latency (DSL), sensory nerve conduction velocity (SCV), sensory nerve action potential (SNAP) wave amplitude were measured. The skin temperature for the examined limbs was maintained at $>32^{\circ} \mathrm{C}$. Reference values were based on the data from healthy individuals in neurophysiology laboratory of Peking Union Medical College Hospital. According to comprehensive evidences of clinical history, neurological symptoms, signs and NCS results, patients were finally diagnosed with or without DPN and subjects of our study were divided into DPN group and non-DPN group.

\section{Laboratory Assay}

Weight and height were determined in subjects wearing light clothing and no shoes by an ultrasonic instrument (Omron HNH-318, Japan). BMI was calculated as body weight in kilograms divided by body height squared in meters $\left(\mathrm{kg} / \mathrm{m}^{2}\right)$.

All patients underwent blood drawing after at least 8 hours of fasting. Fasting plasma glucose (FPG), serum creatinine (SCr), serum uric acid (SUA), serum total 
cholesterol (TC), triglycerides (TG), high-density lipoprotein cholesterol (HDL-C) and low-density lipoprotein cholesterol (LDL-C) were all measured by an autoanalyzer (Roche, Basel, Switzerland). Hemoglobin A1c (HbA1c) was detected by high performance liquid chromatography (Bio-Rad D-10, Berkeley, USA).

The first urine sample in the morning was collected from each subject. Urinary albumin (immunological turbidimetry assay), urinary creatinine (picric acid method), urinary NAG (p-nitrophenol colorimetric method and picric acid method) and urinary $\beta 2-\mathrm{MG}$ (immunological turbidimetry assay) levels were measured by Siemens ADVRI 2400, Germany. The urinary albumin/creatinine ratio (UACR) and urinary NAG/ creatinine ratio $(\mathrm{NAG} / \mathrm{Cr})$ were calculated. The eGFR was calculated using the Chronic Kidney Disease Epidemiology Collaboration (CKD-EPI) equation. ${ }^{10}$ Staging of CKD was classified as G1, G2, G3a, G3b, G4 based on eGFR category. The ranges of eGFR were $\geq 90,60-89,45-59,30-44,15$ $29 \mathrm{~mL} / \mathrm{min} / 1.73 \mathrm{~m}^{2}$, respectively. ${ }^{9}$

\section{Statistical Analysis}

The software SPSS 26.0 was used to calculate the data. The statistical description of the count data was expressed as a rate $(\%)$, and the $\chi^{2}$ test was used for comparison between groups. The measurement data of the normal distribution were represented by mean \pm standard deviation, and the differences between groups were analyzed by independent sample
Student's $t$-test. The non-normal distribution was described as medians (interquartile ranges), and the comparison of differences between groups adopted Kruskal-Wallis $H$ tests in the nonparametric test. At last, DPN was classified as the dependent variable, UACR, eGFR, NAG/Cr and $\beta 2-\mathrm{MG}$ categories (all by tri-sectional quantiles) were independent variables, logistic regression analysis was used to calculate odds ratios and $95 \%$ confidence intervals [OR $(95 \% \mathrm{CI})]$. All models were adjusted for age, sex, BMI, smoking, drinking, hypertension, FPG, HbA1c, TC, TG, HDL-C, LDL-C and duration of diabetes. A test level of $\mathrm{P}<0.05$ was considered statistically significant.

\section{Results}

\section{Comparison of Clinical Characteristics of Study Subjects}

DPN was found in 335 (71.1\%) subjects of all. The age, duration of diabetes, FPG and HbA1c levels were significantly different between DPN group and non-DPN group $(\mathrm{P}<0.05)$. It was shown that subjects in DPN group suffered from older age, longer duration of illness and worse blood glucose control, compared with non-DPN group (Table 1).

\section{The Prevalence of DPN in Different Stages of CKD}

The numbers (proportions) of G1, G2, G3a, G3b, G4 of CKD were $252(53.5 \%), 138$ (29.3\%), 45 (9.6\%), 24

Table I Clinical Characteristics

\begin{tabular}{|c|c|c|c|c|}
\hline Variables & Non-DPN Group $(n=136)$ & DPN Group $(n=335)$ & $x^{2}$ & $\mathbf{P}$ \\
\hline Age (years) & $57.50(52.00-65.00)$ & $63.00(56.00-72.00)$ & & 0.000 \\
\hline Gender ( $\mathrm{n}$, female/male) & $136(76 / 60)$ & $335(176 / 159)$ & 0.435 & 0.510 \\
\hline BMI $\left(\mathrm{kg} / \mathrm{m}^{2}\right)$ & $24.71 \pm 3.22$ & $25.01 \pm 3.63$ & & 0.366 \\
\hline Duration of diabetes (years) & $5.00(1.00-10.00)$ & $8.00(3.00-12.00)$ & & 0.000 \\
\hline Smoking (\%) & 30.1 & 32.2 & 0.196 & 0.658 \\
\hline Drinking (\%) & 9.6 & 10.4 & 0.084 & 0.773 \\
\hline Hypertension (\%) & 52.9 & 56.1 & 0.395 & 0.530 \\
\hline FPG (mmol/L) & $8.03(6.30-10.64)$ & $8.63(6.75-11.72)$ & & 0.026 \\
\hline HbAlc (\%) & $8.10(6.80-10.30)$ & $9.00(7.20-10.90)$ & & 0.023 \\
\hline $\mathrm{BUN}(\mu \mathrm{mol} / \mathrm{L})$ & $5.19(4.23-6.37)$ & $5.40(4.35-6.78)$ & & 0.211 \\
\hline $\mathrm{SCr}(\mu \mathrm{mol} / \mathrm{L})$ & $66.00(54.00-80.75)$ & $69.00(57.00-89.00)$ & & 0.068 \\
\hline $\operatorname{SUA}(\mu \mathrm{mol} / \mathrm{L})$ & $289.00(235.00-355.00)$ & $285.00(235.00-355.00)$ & & 0.952 \\
\hline $\mathrm{TC}(\mathrm{mmol} / \mathrm{L})$ & $4.66(3.8 I-5.50)$ & $4.40(3.76-5.19)$ & & 0.075 \\
\hline TG (mmol/L) & $1.52(1.06-2.53)$ & $1.38(1.00-2.16)$ & & 0.154 \\
\hline HDL-C (mmol/L) & $1.21(1.06-1.43)$ & $1.21(1.06-1.42)$ & & 0.883 \\
\hline LDL-C (mmol/L) & $2.81(2.11-3.38)$ & $2.58(2.02-3.17)$ & & 0.075 \\
\hline
\end{tabular}

Note: Data are presented as mean \pm standard deviation or median (25th-75th percentile) or numbers or proportions.

Abbreviations: DPN, diabetic peripheral neuropathy; BMI, body mass index; FPG, fasting plasma glucose; HbAlc, hemoglobin Alc; SCr, serum creatinine; SUA, serum uric acid; TC, cholesterol; TG, triglycerides; HDL-C, high-density lipoprotein cholesterol; LDL-C, low-density lipoprotein cholesterol. 
(5.1\%) and $12(2.5 \%)$, respectively. Due to the relatively small numbers of member, groups of eGFR $<60 \mathrm{~mL} / \mathrm{min} /$ $1.73 \mathrm{~m}^{2}$ (G3-4) which were considered abnormal were combined $[\mathrm{n}(\%)=81(17.2 \%)]^{10}$

Figure 1 illustrates the prevalence of DPN in G1, G2 and G3-G4. The results suggested that the prevalence of DPN increased gradually with the aggravation of CKD [n $(\%)=167(66.3 \%), 101$ (73.2\%), 67 (82.7\%) respectively, $\mathrm{P}$ value $=0.014]$. The statistical difference was found between $\mathrm{G} 1$ and $\mathrm{G} 3-4(\mathrm{P}=0.005)$.

\section{Differences of Kidney Damage Indicators in Non-DPN Group and DPN Group}

The levels of UACR, NAG/Cr and $\beta 2-\mathrm{MG}$ in DPN group were significantly higher than those in non-DPN group, while the level of eGFR was significantly lower than that in non-DPN group $(\mathrm{P}<0.05)$. (Table 2) It was suggested that the four markers of early renal injury were more serious in DPN group, compared with non-DPN group.

\section{Regression Analysis of Kidney Damage Indexes and DPN}

The values of UACR, eGFR, NAG/Cr and $\beta 2-\mathrm{MG}$ were divided into tri-sectional quantiles. The ranges of each variable are shown in Table 3. DPN was used as the dependent variable, UACR, eGFR, NAG/Cr and $\beta 2-\mathrm{MG}$ categories were used as the independent variables, respectively. The categories of UACR, NAG/Cr and $\beta 2-\mathrm{MG}$ took their lowest tertiles as a reference and the eGFR category took the highest tertiles as a reference.

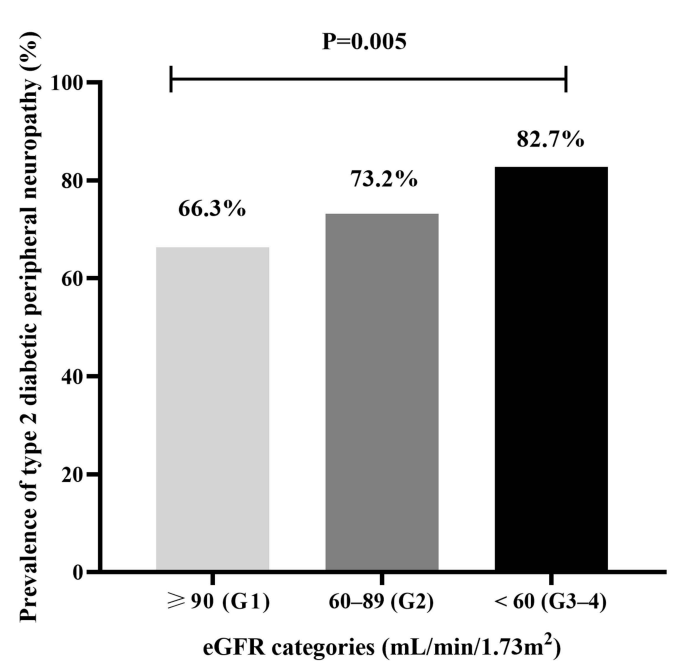

Figure I The prevalence of DPN in different stages of CKD based on eGFR category.
Logistic regression analysis suggested that after adjusting for age, sex, BMI, smoking, drinking, hypertension, FPG, HbAlc, TC, TG, HDL-C, LDL-C and duration of diabetes, $\mathrm{NAG} / \mathrm{Cr}>1.41 \mathrm{U} / \mathrm{mmol}$ (the highest tertile) was a consistently independent risk factor for DPN [OR (95\% $\mathrm{CI})=1.86$ (1.04-3.33), $\mathrm{P}=0.036$ ]. However, UACR, eGFR and $\beta 2-\mathrm{MG}$ did not significantly affect the risk of having DPN $(\mathrm{P}>0.05)$ (see Table 3).

\section{Discussion}

In this study, we found that DPN patients had significantly older age, longer duration of diabetes, higher FPG and HbAlc levels compared with non-DPN patients, which was consistent with the results of previous studies. ${ }^{11}$ It suggests that screening of DPN should pay more attention to the elderly patients with a long duration of diabetes. Good glycemic control is crucial to the prevention and control of DPN, which has been supported by a previous study. ${ }^{12}$

In addition to the above traditional factors, several markers: UACR, eGFR, NAG/Cr and $\beta 2-\mathrm{MG}$ were mainly observed in this study. The most well-known indexes are UACR and eGFR, which reflect glomerular function and are usually used to define staging of CKD. Figure 1 of our results showed that the prevalence of DPN increased gradually with the aggravation of kidney disease. Especially in G3-4, the prevalence of DPN was up to $82.7 \%$. Several studies considered that DKD patients, who were suffered from the overlap of diabetes and CKD, might appear more severe nerve injury, compared with patients of either diabetes or CKD alone. ${ }^{5,13}$ On the one hand, insulin resistance induced by diabetes component might lead to an inability response of peripheral nerve to the trophic support of insulin. ${ }^{14}$ On the other hand, axonal dysfunction caused by CKD component played a critical role in the development of DPN. ${ }^{5}$ Besides, higher UACR and lower eGFR levels were found in DPN group than those in nonDPN group. A prospective study ${ }^{15}$ got the cut-off values of eGFR $\left(<65.3 \mathrm{~mL} / \mathrm{min} / 1.73 \mathrm{~m}^{2}\right)$ and UACR $(>98.6 \mathrm{mg} / \mathrm{dl})$ to be cautious of the presence of DPN, even in asymptomatic patients. Another large sample study ${ }^{16}$ of 30,423 T2DM patients considered that in earlier stage of kidney disease (G3), the microvascular disease such as endothelial dysfunction was involved in the development of polyneuropathy. The advanced stage (G4-5) was more strongly associated with polyneuropathy by multiple factors including uremic toxins effects, abnormal membrane excitability induced by an inhibitory effect on the activity of the axonal Na-K pump, increased activity of the $\mathrm{Na}^{+} / \mathrm{Ca}^{2+}$ 
Table 2 Comparison of Kidney Damage Indicators

\begin{tabular}{|c|c|c|c|}
\hline Variables & Non-DPN Group & DPN Group & $\mathbf{P}$ \\
\hline UACR (mg/g) & $15.54(9.53-36.89)$ & $25.20(11.95-101.00)$ & 0.001 \\
\hline eGFR $\left(\mathrm{mL} / \mathrm{min} / 1.73 \mathrm{~m}^{2}\right)$ & $97.82(79.85-106.60)$ & $89.91(64.42-100.59)$ & 0.000 \\
\hline $\mathrm{NAG} / \mathrm{Cr}(\mathrm{U} / \mathrm{mmol})$ & $0.82(0.55-I .4 I)$ & I.I4 (0.70-I.70) & 0.001 \\
\hline ß2-MG (mg/L) & $0.08(0.03-0.24)$ & $0.12(0.04-0.57)$ & 0.028 \\
\hline
\end{tabular}

Note: Data are presented as median (25th-75th percentile).

Abbreviations: UACR, urinary albumin/creatinine ratio; eGFR, estimated glomerular filtration rate; NAG/Cr, urinary $\mathrm{N}$-acetyl- $\beta$-D-glucosaminidase/creatinine ratio; $\beta 2-M G$, urinary $\beta 2$ microglobulin.

Table 3 Risk Factors for Having DPN

\begin{tabular}{|c|c|c|c|c|}
\hline Variables & Categories & Ranges & OR (95\% Cl) & $\mathbf{P}$ \\
\hline \multirow[t]{3}{*}{ UACR (mg/g) } & I & $\leq|2.8|$ & I & - \\
\hline & 2 & | $2.82-38.20$ & I.06 (0.62-I.79) & 0.835 \\
\hline & 3 & $>38.20$ & $1.66(0.92-3.01)$ & 0.092 \\
\hline \multirow[t]{3}{*}{ eGFR $\left(\mathrm{mL} / \mathrm{min} / 1.73 \mathrm{~m}^{2}\right)$} & I & $>99.01$ & I & - \\
\hline & 2 & $80.77-99.01$ & $1.59(0.88-2.88)$ & 0.124 \\
\hline & 3 & $\leq 80.76$ & $1.37(0.73-2.59)$ & 0.331 \\
\hline \multirow[t]{3}{*}{$\mathrm{NAG} / \mathrm{Cr}(\mathrm{U} / \mathrm{mmol})$} & I & $\leq 0.75$ & 1 & - \\
\hline & 2 & $0.76-1.41$ & I.46 (0.86-2.47) & 0.162 \\
\hline & 3 & $>1.4 \mathrm{I}$ & $1.86(1.04-3.33)$ & 0.036 \\
\hline \multirow[t]{3}{*}{$\beta 2-M G$ (mg/L) } & I & $\leq 0.04$ & 1 & - \\
\hline & 2 & $0.05-0.24$ & $0.88(0.52-1.47)$ & 0.616 \\
\hline & 3 & $>0.24$ & I.7I (0.94-3.08) & 0.077 \\
\hline
\end{tabular}

Note: All models were adjusted for age, sex, BMI, smoking, drinking, hypertension, FPG, HbAIc, TC, TG, HDL-C, LDL-C and duration of diabetes.

exchanger, endothelial injury, oxidative stress, neurohormonal abnormality and inflammation.

Previous studies have mostly evaluated the relationship between markers of glomerular injury and DPN, possibly because they are signs for staging of kidney disease. Actually, renal tubular injury is also an important component in the development of DKD. Firstly, a number of studies have indicated that tubular dysfunction may precede glomerular damage in DKD. ${ }^{17}$ Secondly, biomarkers of tubular injury can provide critical information when serum creatinine does not change appreciably. In other words, biomarkers of tubular injury have great potentiality in prognosticating the development of incident chronic kidney disease. ${ }^{18}$ Urinary NAG and $\beta 2-\mathrm{MG}$ are typical biomarkers of renal tubular function. ${ }^{19}$ NAG is a lysosomal brush-margin enzyme located in the microvilli of renal tubule epithelial cells. Due to its relatively high molecular weight, NAG cannot be filtered through the glomeruli and is only released into the urine after renal tubule injury. $\beta 2-\mathrm{MG}$ is a low-molecular-weight protein that is filtered by the glomeruli and then almost completely reabsorbed and catabolized by the cells of the proximal tubule. In our study, we further found that levels of NAG/ $\mathrm{Cr}$ and $\beta 2-\mathrm{MG}$ had an interesting increase in DPN group than those in non-DPN group. There were few studies on the relationship between renal tubular injury and DPN. Hong et $\mathrm{al}^{17}$ showed that urinary excretion of tubular proteins (RBP and $\beta 2-\mathrm{MG}$ ) and tubular enzyme (total NAG) were higher in diabetes patients with microvascular complications (retinopathy and peripheral neuropathy). The possible explanation was that the development of tubular dysfunction and other microvascular complications depended on a common pathogenic factor, such as metabolic dysfunction. ${ }^{17,20}$ Other mechanism mentioned that $\beta 2-\mathrm{MG}$, one of the uremic toxins, could indirectly cause neurotoxin effect. ${ }^{16}$ The toxicity of $\beta 2-\mathrm{MG}$ is strongly amplified by an environment of inflammation and oxidative stress $^{21}$ which are also the pathological process of DPN.

The findings of this study pointed out that significant differences were found in each renal indexes between DPN group and non-DPN group; however, NAG/Cr 
appeared to be the only marker with the advantage of predicting DPN. The highest tertile of NAG/Cr $(>1.41 \mathrm{U} /$ mmol) was an independent risk factor for DPN, while no association was observed for other indicators. In the past, Kopf et al proposed for the first time that urinary NAG excretion was an independent marker of DPN and cardiovascular autonomic neuropathy through a cross-sectional study. ${ }^{22}$ However, the specific mechanism between NAG and DPN remains unclear. Toshihiro Takao et al suggested that urinary NAG excretion was associated with NF- $\mathrm{NB}$ activation. ${ }^{23} \mathrm{NF}-\mathrm{kB}$ is a key transcription factor in inflammation and immunity and can promote the development of inflammation through a variety of mechanisms, including abnormal survival of autoreactive B cells, production of autoimmune antibodies, recruitment of abnormal chemokine and inflammatory cells. ${ }^{24}$ Meanwhile, inflammation is closely involved in the development of DPN. We boldly hypothesize a possible pathological mechanism for our results, that is, urinary NAG excretion triggers NF- $\mathrm{KB}$ activation, which further promotes inflammation and affects the development of DPN. More researches are needed to confirm this hypothesis.

Based on the results, we did not believe that other markers of renal injury were no longer clinically significant. At first, the four markers reflected renal damage and/ or progression of kidney disease. In addition to early prediction of DKD, these indexes could also be considered for prediction of DPN. It was possible that the phenotype of NAG/Cr might appear earlier and was more sensitive in DPN than others. This was similar to the view that tubular injury might precede glomerular injury in DKD. Secondly, the unified mechanism theory of diabetic complications suggests that hyperglycemia selectively damages a particular subset of cell types: capillary endothelial cells in the retina, mesangial cells in the renal glomerulus, and neurons and Schwann cells in peripheral nerves. A consistent differentiating feature common to all cell types, which are damaged by hyperglycemia, is an increased production of reactive oxygen species (ROS). ${ }^{25}$ Therefore, DKD and DPN can be considered to have a unified pathogenesis and the indexes of renal injury are potentially effective for nerve injury.

Strengths of this study include that we innovatively combined some early markers of glomerular and tubular injury and evaluated the role of these markers for early screening of DPN for the first time. The clinical significance of this study is to guard against the potential DPN without positive symptoms, based on easily available urinary sample detection. There are some limitations. Firstly, selection bias could not be avoided because only hospitalized patients were included. Secondly, our sample size was limited. A prospective study with a larger sample size and longterm follow-up may provide more cause-effect conclusions. In addition, some specific pathological mechanisms between NAG and DPN are unclear and need to be further studied.

\section{Conclusions}

When T2DM patients suffer from CKD, DPN will be more likely to appear, accelerate or deteriorate due to dual pathological exposure. Some easily available urinary markers of glomerular and tubular damage, such as UACR, eGFR, NAG/Cr and $\beta 2-\mathrm{MG}$, can be used for early prediction of DPN, in which increased NAG/Cr is an independent risk factor for DPN.

\section{Data Sharing Statement}

All data in this study are available from the corresponding author upon reasonable request.

\section{Ethics Approval and Consent to Participate}

The study protocol was approved by the ethics committee of the Third Hospital of Nanchang. The written informed consent was obtained from each subject. The study was carried out in conformity to the Declaration of Helsinki (as revised in 2013).

\section{Author Contributions}

All authors made a significant contribution to the work reported, whether that is in the conception, study design, execution, acquisition of data, analysis and interpretation, or in all these areas; took part in drafting, revising or critically reviewing the article; gave final approval of the version to be published; have agreed on the journal to which the article has been submitted; and agree to be accountable for all aspects of the work.

\section{Funding}

This work was supported by the National Natural Science Foundation of China (grant number 81760153, Recipient: Peng Duan), the Key Research and Development Programs by Science and Technology Department of Jiangxi Province (grant numbers 20171BBG70058, 20171ACH80002 and 20181BBG70014, Recipient: Zhi Yang, Ping $\mathrm{Tu}$ and Jiang Liu), the Science and Technology Support Project by Science and Technology 
Department of Nanchang City (grant number [2020]133, Recipient: Peng Duan).

\section{Disclosure}

The authors declare that they have no competing interests.

\section{References}

1. Zheng Y, Ley SH, Hu FB. Global aetiology and epidemiology of type 2 diabetes mellitus and its complications. Nat Rev Endocrinol. 2018;14(2):88-98. doi:10.1038/nrendo.2017.151

2. Pop-Busui R, Boulton AJ, Feldman EL, et al. Diabetic neuropathy: a position statement by the American Diabetes Association. Diabetes Care. 2017;40(1):136-154. doi:10.2337/dc16-2042

3. American Diabetes Association. 11. microvascular complications and foot care: standards of medical care in diabetes-2021. Diabetes Care 2021;44(Supplement 1):S151-S167. doi:10.2337/dc21-S011

4. Aggarwal HK, Sood S, Jain D, et al. Evaluation of spectrum of peripheral neuropathy in predialysis patients with chronic kidney disease. Ren Fail. 2013;35(10):1323-1329. doi:10.3109/ 0886022X.2013.828261

5. Issar T, Arnold R, Kwai NCG, et al. Relative contributions of diabetes and chronic kidney disease to neuropathy development in diabetic nephropathy patients. Clin Neurophysiol. 2019;130 (11):2088-2095. doi:10.1016/j.clinph.2019.08.005

6. Zhang Y, Jiang Y, Shen X, et al. Can both normal and mildly abnormal albuminuria and glomerular filtration rate be a danger signal for diabetic peripheral neuropathy in type 2 diabetes mellitus? Neurol Sci. 2017;38(8):1381-1390. doi:10.1007/s10072017-2946-1

7. Kim SR, Lee YH, Lee SG, et al. Urinary N-acetyl-beta -D-glucosaminidase, an early marker of diabetic kidney disease, might reflect glucose excursion in patients with type 2 diabetes. Medicine. 2016;95(27):e4114. doi:10.1097/MD.0000000000004114

8. Ekrikpo UE, Effa EE, Akpan EE, et al. Clinical utility of urinary beta 2-microglobulin in detection of early nephropathy in African diabetes mellitus patients. Int $J$ Nephrol. 2017;2017:4093171. doi:10.1155/ 2017/4093171

9. Levey AS, Coresh J, Balk E, et al.; National Kidney Foundation. National Kidney Foundation practice guidelines for chronic kidney disease: evaluation, classification, and stratification. Ann Intern Med. 2003;139(2):137-147. doi:10.7326/0003-4819-139-2-20030715000013

10. National Kidney Foundation. KDIGO 2012 clinical practice guideline for the evaluation and management of chronic kidney disease. Kidney Int Suppl. 2013;3:1-150.

11. Liu X, Xu Y, An M, et al. The risk factors for diabetic peripheral neuropathy: a meta-analysis. PLoS One. 2019;14(2):e212574.
12. Wu F, Jing $\mathrm{Y}$, Tang $\mathrm{X}$, et al. Anemia: an independent risk factor of diabetic peripheral neuropathy in type 2 diabetic patients. Acta Diabetol. 2017;54(10):925-931. doi:10.1007/s00592-017-1025-7

13. Jasti DB, Mallipeddi S, Apparao A, et al. A clinical and electrophysiological study of peripheral neuropathies in predialysis chronic kidney disease patients and relation of severity of peripheral neuropathy with degree of renal failure. J Neurosci Rural Pract. 2017;8 (4):516-524. doi:10.4103/jnrp.jnrp_186_17

14. Feldman EL, Nave KA, Jensen TS, et al. New horizons in diabetic neuropathy: mechanisms, bioenergetics, and pain. Neuron. 2017;93 (6):1296-1313. doi:10.1016/j.neuron.2017.02.005

15. Lai YR, Cheng BC, Huang CC, et al. Correlation between kidney and peripheral nerve functions in type 2 diabetes. QJM. 2020;113 (3): $173-180$

16. Kaewput W, Thongprayoon C, Rangsin R, et al. The association between renal function and neurological diseases in type 2 diabetes: a multicenter nationwide cross-sectional study. Hosp Pract. 2019;47 (1):46-52. doi:10.1080/21548331.2019.1549916

17. Hong CY, Chia KS, Ling SL. Urinary protein excretion in type 2 diabetes with complications. J Diabetes Complications. 2000;14 (5):259-265. doi:10.1016/S1056-8727(00)00119-7

18. Zhang WR, Parikh CR. Biomarkers of acute and chronic kidney disease. Annu Rev Physiol. 2019;81(1):309-333. doi:10.1146/ annurev-physiol-020518-114605

19. Mise K, Hoshino J, Ueno T, et al. Prognostic value of tubulointerstitial lesions, urinary $\mathrm{N}$-acetyl-beta-d-glucosaminidase, and urinary beta2-microglobulin in patients with type 2 diabetes and biopsy-proven diabetic nephropathy. Clin J Am Soc Nephrol. 2016;11(4):593-601. doi:10.2215/CJN.04980515

20. Holm J, Nielsen NV, Hemmingsen L. Retinopathy in type II diabetes mellitus associated with above-normal urinary excretion of RBP. Kidney Int Suppl. 1994;47:S105-S108.

21. Canaud B, Morena M, Cristol JP, Krieter D. Beta2-microglobulin, a uremic toxin with a double meaning. Kidney Int. 2006;69 (8):1297-1299. doi:10.1038/sj.ki.5000389

22. Kopf S, Oikonomou D, Zdunek D, et al. Urinary n-acetyl-beta-d-glucosaminidase excretion: an indicator of neuropathy in type 2 diabetes. Exp Clin Endocrinol Diabetes. 2013;121(10):601-606. doi:10.1055/ s-0033-1355384

23. Takao T, Horino T, Kagawa T, et al. Possible involvement of intracellular angiotensin II receptor in high-glucose-induced damage in renal proximal tubular cells. $J$ Nephrol. 2011;24(2):218-224. doi: $10.5301 / \mathrm{JN} .2010 .5785$

24. Sun SC. The non-canonical NF-kappaB pathway in immunity and inflammation. Nat Rev Immunol. 2017;17(9):545-558. doi:10.1038/ nri. 2017.52

25. Brownlee M. The pathobiology of diabetic complications: a unifying mechanism. Diabetes. 2005;54(6):1615-1625. doi:10.2337/ diabetes.54.6.1615

\section{Publish your work in this journal}

Diabetes, Metabolic Syndrome and Obesity: Targets and Therapy is an international, peer-reviewed open-access journal committed to the rapid publication of the latest laboratory and clinical findings in the fields of diabetes, metabolic syndrome and obesity research. Original research, review, case reports, hypothesis formation, expert opinion and commentaries are all considered for publication. The manuscript management system is completely online and includes a very quick and fair peer-review system, which is all easy to use. Visit http://www.dovepress.com/testimonials.php to read real quotes from published authors. 